Published in final edited form as:

Psychol Sci. 2014 February 1; 25(2): 466-475. doi:10.1177/0956797613509284.

\title{
Reward-based Transfer from Bottom-up to Top-down Search Tasks
}

\author{
Jeongmi Lee and Sarah Shomstein \\ Department of Psychology, The George Washington University
}

\begin{abstract}
Recent evidence suggests that reward modulates bottom-up and top-down attentional selection, persisting within the same task even when reward is no longer offered. It remains unclear whether reward transfer occurs across tasks, especially those engaging different modes of attention. We directly investigate whether reward-based contingency learned in a bottom-up search task is transferred to a subsequent top-down search task, and probe the nature of the transfer mechanism. Results showed that reward benefit established in a pop-out search task was transferred to a conjunction search task, increasing search efficiency for targets previously associated with higher reward. Reward history influenced search efficiency by both enhancing target salience and distractor filtering, depending on whether the target and distractors share a critical feature. These results provide evidence for reward-based transfer between different modes of attention, and strongly suggest that an integrated priority map, based on reward information, guides both topdown and bottom-up attention.
\end{abstract}

\section{Keywords}

Attention; Rewards; Visual Search

Efficiently allocating attentional resources to a subset of relevant information is crucial for increasing fitness of an observer. Research demonstrated that allocation of attention is controlled by two partially segregated networks of brain areas. Top-down attention system, recruiting parts of the intraparietal and superior frontal cortices, is specialized for selecting stimuli based on cognitive factors, such as current goals and expectations. Bottom-up attention system, on the other hand, recruits the temporoparietal and inferior frontal cortices, and is involved in processing stimuli based on stimulus-driven factors, such as physical salience and novelty (Corbetta \& Shulman, 2002; Kastner \& Ungerleider, 2000; Shomstein, Lee, \& Behrmann, 2010).

Another important contributor to increasing fitness is evaluating reward contingencies and adjusting behaviors accordingly. Recent evidence indicates that reward is a powerful determinant of attentional deployment. Studies showed that top-down attentional priority is given to stimuli associated with higher probability or amounts of reward (Della Libera \& Chelazzi, 2006; Lee \& Shomstein, 2013; Navalpakkam, Koch, Rangel, \& Perona, 2010; Shomstein \& Johnson, in press). Reward-based modulation is also applied to bottom-up attention, evidenced by studies showing that even the most efficient pop-out search is

Correspondence should be addressed to Jeongmi Lee, Department of Psychology, George Washington University, 2125 G Street NW, Washington, DC 20052. jeongmi@gwmail.gwu.edu; Phone: 2029948725.

Authorship

J. Lee and S. Shomstein designed the study. Testing, data collection, and data analysis were performed by J. Lee under the supervision of S. Shomstein. J. Lee and S. Shomstein wrote the paper. All authors approved the final version of the paper for submission. 
modulated by reward schedules (Kiss, Driver, \& Eimer, 2009; Kristjansson, Sigurjonsdottir, $\&$ Driver, 2010). Interestingly, effects of reward persist when the reward contingency is no longer relevant, even several days after training (Anderson, Laurent, \& Yantis, 2011b; Della Libera \& Chelazzi, 2009).

Although it is well established that attention is strongly modulated by reward and the effects persist within the same type of task, it remains unclear whether the effect of reward contingency learned within one type of task is transferred to a different type of task, specifically when the two tasks engage different attentional orienting systems, top-down and bottom-up. If effects of reward transfer across different attentional systems, then it would suggest that an integrated priority map, based on reward contingencies, guides attentional selection. Alternatively, if the transfer does not occur, it would suggest that reward-based priority maps are derived separately for top-down and bottom-up selection.

Considering that in real world environments reward-associated stimuli appear in dynamic contexts combined with various features, it is critical to understand how a rewarded feature is integrated with other features and whether the effects are generalized across different attentional settings. Most previous studies, however, focused exclusively on the effect of reward within a single attentional domain (i.e., top-down or bottom-up), investigating the lingering effects of reward contingency in the similar type of tasks with no reward schedule (Della Libera \& Chelazzi, 2009), or the effect of reversing reward schedules within the identical task (Kristjansson, et al., 2010). A recent study (Anderson, Laurent, \& Yantis, 2011a) demonstrated value-based transfer from top-down to bottom-up tasks, in which attentional capture by a distractor was modulated by its prior reward association. However, while important, the results are not sufficient to interpret as providing direct evidence for an integrated reward-based priority map. First, the same reward-associated stimuli were used in both top-down and bottom-up tasks (e.g., a red circle), which did not allow for examining integration of a rewarded feature with other features. Second, the target feature associated with reward in the training phase was used as a distractor feature in the test phase, making it unclear whether the observed transfer was due to a lingering target template or the effect of prior reward associations. Third, the results only provided evidence for a unidirectional transfer (from top-down to bottom-up), in which the effect could be due to lingering conscious valuation developed in the top-down phase. Therefore, for conclusive evidence for an integrated reward-based priority map, it is necessary to demonstrate transfer effect in the opposite direction, i.e., from bottom-up to top-down.

Here, we trained reward contingency in a pop-out search task using a biased reward schedule, and tested whether the effects of reward are transferred to a subsequent conjunction search task with no biased reward schedule. This way, we could demonstrate how a rewarded feature is integrated with other features and whether the effects are generalized across different attentional settings. Additionally, we examined whether the salience in the priority map is determined by enhancing target features previously associated with reward or by suppressing distractor features, or both.

\section{Methods}

\section{Participants}

Different groups of undergraduate students ranging in age from 18 to 22 participated in onehour session [Experiment 1: 22 (14 female), Experiment 2: 19 (11 female), Experiment 3: 25 (14 female)]. All had normal or corrected-to-normal vision and gave informed consent. All of the experimental procedures were approved by the Institutional Review Board of the George Washington University. 


\section{Task}

In the pop-out search task (Fig. 1a), each trial included a sequence of fixation, search display, and feedback. The fixation display consisted of a white fixation cross $\left(0.5^{\circ} \times 0.5^{\circ}\right)$ placed in the center of a black background for 700ms. A search display, comprised of white line segments $\left(1.13^{\circ}\right.$ in length), remained visible until response. The set size on each trial varied randomly among 9,16 , and 25 , and the line segments were arranged in a matrix of $3 \times 3,4 \times 4$, or $5 \times 5$, separated by $1.5^{\circ}$ from each other. The target was defined by a unique orientation, either horizontal or vertical, randomly positioned among $45^{\circ}$ oriented distractors. Participants were asked to press " 1 " key for horizontal targets, and to press " 2 " key for vertical targets. Each response was followed by reward feedback for $800 \mathrm{~ms}$. One of the target orientations (horizontal, vertical) was more highly rewarded than the other, counterbalanced across participants. High reward targets were followed by high reward feedback ( 10 points) on $80 \%$ of trials and low reward feedback (1 point) on the remaining $20 \%$, and vice versa. Five points were deducted for incorrect responses.

For the conjunction search task (Fig. 1b), Experiment 2 and 3 were slightly different from Experiment 1 (explained in the corresponding sections). In Experiment 1, a white fixation cross $\left(0.5^{\circ} \times 0.5^{\circ}\right)$ was presented for $700 \mathrm{~ms}$, followed by a target prime in which words "Red horizontal" or "Red vertical" were presented centrally in white color (font size 18) for $1,000 \mathrm{~ms}$. A search display consisted of line segments ( $1.13^{\circ}$ in length) of various colors and orientations, remained visible until response. The arrangement and set size followed the same restrictions as in the pop-out search task. The target was defined by a combination of color and orientation, either red horizontal or red vertical, embedded in distractors that had combinations of four different colors (blue, green, orange, purple) and orientations ( $30^{\circ}$, $60^{\circ}, 120^{\circ}, 150^{\circ}$ ). Half of the distractors shared a color feature with the target and the other half of the distractors shared an orientation feature with the target. Seventy percent of trials were target-present trials. Participants were asked to press "c" key for target-present trials, and "m" key for target-absent trials. After each response, a 700ms feedback display indicated whether the response was correct or incorrect with no reward feedback, nullifying the reward associations learned in the pop-out search task.

Initial set of 200 trials of conjunction search task was performed to obtain baseline performance before exposure to biased-reward training. Then, a block of pop-out search task (50 trials) alternated with a block of conjunction search task (50 trials) five times. For motivation, participants were told that they would get 2 dollars on top of a course credit for accumulating more than $95 \%$ of the total points. In fact, all participants were paid $\$ 2$ on top of a course credit on completion of the experiment, and were fully debriefed.

\section{Results}

\section{Experiment 1}

We tested whether the effects of reward established in the context of bottom-up search are transferred to a different context in which top-down search is required, even when the reward contingency is no longer relevant. To rule out the possibility that the reward-based carryover effect is due to motor enhancement rather than sensory enhancement, participants responded to different aspects of the target (orientation for pop-out search, and presence for conjunction search) with different key mappings.

For the pop-out search task (Fig. 2a), a repeated measures analysis of variance (ANOVA) with reward (high, low) and set size $(9,16,25)$ as within subject factors and reaction times (RTs) as the dependent measure revealed a significant main effect of reward $[\mathrm{F}(1$, $21)=40.14, p<.001]$, with faster mean RTs for high reward targets $(648 \mathrm{~ms})$ than for low 
reward targets $(692 \mathrm{~ms})$. This validated that reward manipulation elicits a significant modulation effect in favor of the target orientation associated with higher reward. A set size effect was also significant $[\mathrm{F}(2,42)=4.16, p<.05]$, with faster mean RTs for set size 16 (662ms) than set size $25(676 \mathrm{~ms})[\mathrm{F}(1,21)=7.83, p<.05]$. Although the set size effect is unexpected considering previous results that RTs for pop-out search mostly stay flat across set sizes (Treisman \& Gelade, 1980; Wolfe, 1994), it can be explained by grouping effect that diagonal distractors are easily grouped together for set size 16, making search more efficient (Humphreys, Quinlan, \& Riddoch, 1989; Nothdurft, 1993). The reward by set size interaction was not significant. Mean accuracy rate was $97 \%$, with no significant main effects or interaction.

For the conjunction search task, trials were classified as containing a previously high- or low-rewarded target based solely on the reward contingencies in the pop-out search task. Central to the study was the performance in target-present trials. A repeated measures ANOVA (Fig. $2 b)$ revealed a significant reward effect $[\mathrm{F}(1,21)=20.57, p<.001]$, with faster mean RTs for previously high-rewarded targets $(934 \mathrm{~ms})$ than for previously low-rewarded targets $(997 \mathrm{~ms})$. A set size effect was also significant $[\mathrm{F}(2,42)=144.12, p<.001]$, with RTs linearly increasing as the set size increased, confirming that the task involved attentionally demanding top-down search. Most importantly, the reward by set size interaction was significant $[\mathrm{F}(2,42)=15.78, p<.001]$, indicating that search efficiency for targets differed based on the prior associations with reward. To compare search efficiency, search slopes were calculated for each condition by dividing RT differences by set size differences. Search for targets previously associated with higher reward was more efficient, evidenced by a shallower search slope for previously high-rewarded targets $(19 \mathrm{~ms} / \mathrm{item})$ than for previously low-rewarded targets $(27 \mathrm{~ms} / \mathrm{item})$. Accuracy rate results were consistent with the pattern observed in the RT data, with no speed-accuracy trade-offs (supplementary material).

These results are important for two reasons. First, they corroborate previous findings showing that even the most efficient bottom-up search is modulated by reward (Kiss, et al., 2009; Kristjansson, et al., 2010). Secondly, and more importantly, we demonstrate a novel finding that the effects of reward established within a bottom-up search task are transferred to a subsequent top-down search task, even when the reward contingency is no longer relevant and the previously rewarded feature is integrated with other features.

To examine how conjunction search performance is affected by reward-based transfer effects, we compared baseline conjunction search performance before reward training with that after reward training, investigating whether search becomes more efficient for previously high-rewarded targets, or less efficient for previously low-reward targets, or both. Since there was no significant difference $(\mathrm{F}<1)$ in baseline conjunction performance for tobe high- and to-be-low-rewarded targets, baseline data were collapsed across that factor. For target-present trials, a repeated measures ANOVA was conducted with session (baseline, post-reward training) and set size $(9,16,25)$ as within subject factors and RTs as the dependent measure, separately for previously high- and low-rewarded targets. A session effect was observed for both previously high- $[\mathrm{F}(1,21)=54.76, p<.001]$ and low-reward targets $[\mathrm{F}(1,21)=20.92, p<.001]$, with faster mean RTs for post-reward training (934ms for previously high-rewarded targets, $997 \mathrm{~ms}$ for previously low-rewarded targets) than that of baseline $(1091 \mathrm{~ms})$, possibly reflecting practice effects. The set size effect was also significant for both previously high- $[\mathrm{F}(2,42)=212.98, p<.001]$ and low-rewarded targets $[\mathrm{F}(1,21)=287.21, p<.001]$, with linearly increasing RTs across set sizes. More importantly, for previously high-rewarded targets, the session by set size interaction was significant $[\mathrm{F}(2$, $42)=4.70, p<.05]$. Search for previously high-rewarded targets became more efficient after reward training (19ms/item) than baseline (24ms/item) (Fig. 2c), while search efficiency for previously low-rewarded targets remained unchanged relative to baseline. These results 
demonstrate that reward-based transfer occurs exclusively for previously high-rewarded targets, and not for previously low-rewarded targets.

Next, we further hypothesized that if change in search efficiency in the conjunction search task is a direct result of reward-based transfer from the pop-out search task, then participants with greater reward effects in the pop-out search task should exhibit greater amount of reward-based transfer in the conjunction search task. The direct relationship was examined by correlating the magnitude of reward effects in the pop-out search task with that in the conjunction search task. The magnitude of reward effects was calculated for each participant by averaging RT differences between high and low reward targets in each set size. There was a significant positive correlation between the magnitude of reward effects in the two tasks ( $\mathrm{r}=.45, p<.05)$ (Fig. $2 d$ ), indicating that the degree of reward-based benefit in bottomup search predicts the extent of reward-based carry-over in the subsequent top-down search. This result provides direct evidence that the effects of reward contingency learned in the context of bottom search are transferred to a different context in which reward contingency is irrelevant and top-down search is required.

\section{Experiment 2}

Experiment 1 examined whether search efficiency is modulated by a target feature previously associated with reward. Experiment 2 focused on how search efficiency is modulated by the presence of a distractor feature previously associated with reward. Namely, we asked whether distractors previously associated with reward enhance search efficiency via increased distractor filtering, or hinder search by capturing attention. For that purpose, distractors that contain a feature previously associated with reward were included in the conjunction search display. Half of the target-colored (red) distractors had an orientation that was previously associated with reward (vertical for a red horizontal target, horizontal for a red vertical target), and the other half of the red distractors had neutral orientations $\left(30^{\circ}, 60^{\circ}, 120^{\circ}\right.$, or $\left.150^{\circ}\right)$ (Fig. $3 a$ ). By comparing conjunction search performance in Experiment 2 (a mix of 'loaded' and neutral distractors) with that in Experiment 1 (neutral distractors), we tested whether adding previously rewarded distractors enhances or hinders search performance. If loaded distractors are filtered out more efficiently, search efficiency should be greater in Experiment 2 than in Experiment 1. On the other hand, if loaded distractors capture attention, search should be less efficient in Experiment 2 than in Experiment 1.

We replicated Experiment 1, such that pop-out search was modulated by reward (Fig. 3b) and the modulation effect was transferred to the subsequent conjunction search (Fig. $3 c$; see supplementary material). Next, we examined the effect of the presence of previously rewarded distractors on conjunction search efficiency. Since the relationship between target and distractor orientations is different between Experiment 1 and 2, we normalized the RT data in each experiment by subtracting RT in post-reward training blocks from the RT in baseline blocks for each subject, reward condition, and set size. The resulted RT differences from baseline reflect the pure effect of reward regardless of target-distractor contexts, since the physical stimuli were exactly the same between baseline and post-reward training blocks. Then, we calculated the slope of the RT differences to quantify the effect of reward on conjunction search efficiency (Fig. 3d). Greater slope of the RT differences indicate greater reward effects on the conjunction search efficiency as compared to baseline.

For target-present trials, a mixed ANOVA was conducted with the experiment $(1,2)$ as a between subject factor, reward (high, low) as a within subject factor, and the slope of the RT differences from baseline as the dependent measure (Fig. $3 d$ ). There was a significant reward effect $[\mathrm{F}(1,39)=30.54, p<.001]$, with greater mean slope differences for previously high-rewarded targets $(6.7 \mathrm{~ms} / \mathrm{item})$ than for previously low-rewarded targets $(0.6 \mathrm{~ms} /$ item $)$. 
Most importantly, the main effect of experiment was significant $[\mathrm{F}(1,39)=6.53, p<.05]$, with greater mean slope differences in Experiment $2(6.4 \mathrm{~ms} / \mathrm{item})$ than in Experiment $1(1.0 \mathrm{~ms} /$ item). The reward by experiment interaction was not significant, indicating that the presence of previously rewarded distractors enhanced search efficiency regardless of reward levels. This result provides strong evidence that search becomes more efficient when targets are embedded among distractors previously associated with reward, suggesting that the benefit of reward-based transfer is not confined to target features, but also extends to distractor features, making search more efficient via increased distractor filtering.

\section{Experiment 3}

Experiment 2 demonstrated that the presence of a previously rewarded feature improves search efficiency by increasing distractor filtering. This result is somewhat counterintuitive, since previous studies demonstrated that a distractor previously associated with reward hinders search by capturing attention (Anderson, et al., 2011a, 2011b). One of the possible explanations for the contrasting findings is that previously rewarded distractors in Experiment 2 were filtered out more efficiently because they shared a critical feature (color) with the target. The target in the conjunction search task was either red horizontal or red vertical line, making the red color as a critical feature in setting a target template (Folk, Remington, \& Johnston, 1992). Also, a target prime presented in each trial could have helped setting a target template in advance, selectively enhancing the target orientation and suppressing the distractor orientation. To address this possibility, in Experiment 3, a target prime was not presented, eliminating an advance set up of a target template. Also, previously rewarded distractors shared an orientation feature with the target, instead of sharing a color feature. Nontarget-colored distractors had an orientation previously associated with reward (high, low) or had a neutral orientation $\left(135^{\circ}\right)$. The reward levels of target (high, low) and distractors (high, low, neutral) were fully crossed and trial types were randomly intermixed.

For the pop-out search task (Fig. 4a), the same pattern of result was replicated (supplementary material). For the conjunction search task (Fig. 4b, supplemental material), search slope for each condition was calculated from RTs and submitted to a repeated measures ANOVA, with target reward (high, low) and distractor reward (high, low, neutral) as within subject factors and search slopes as the dependent measure (Fig. $4 c$ ). There was a significant target reward effect $[\mathrm{F}(1,24)=30.42, p<.001]$, with shallower mean search slopes for previously high-rewarded targets $(20 \mathrm{~ms} / \mathrm{item})$ than for previously low-rewarded targets $(28 \mathrm{~ms} /$ item $)$. The distractor reward effect was also significant $[\mathrm{F}(2,48)=6.89, p<.005]$, with shallower mean search slopes among neutral distractors $(20 \mathrm{~ms} / \mathrm{item})$ than either among previously high-rewarded $(26 \mathrm{~ms} / \mathrm{item})$ or previously low-rewarded $(25 \mathrm{~ms} / \mathrm{item})$ distractors, suggesting that previously rewarded distractors interfered with search, compared with neutral distractors. More importantly, target reward by distractor reward interaction was significant $[\mathrm{F}(2.48)=7.22, p<.005]$, indicating that search efficiency changed based on the target and distractor's previous reward associations. For previously high-rewarded targets, search among previously high-rewarded distractors $(18 \mathrm{~ms} / \mathrm{item})$ was as efficient as search among neutral distractors (18ms/item), with previously low-rewarded distractors (23ms/ item) significantly interfering search. For previously low-rewarded targets, on the other hand, both previously high- and low-rewarded distractors interfered with search as compared to neutral distractors $(21 \mathrm{~ms} /$ item $)$, with greater interference from previously high(35ms/item) than low- $(28 \mathrm{~ms} /$ item) rewarded distractors. These results suggest that the extent of distractor interference is dependent on the current target template: when searching for a previously high-rewarded target, it is easier to selectively suppress previously highrewarded distractors. When searching for a previously low-rewarded target, on the other 
hand, it is hard to suppress distractor features, especially the ones previously associated with high reward.

To examine a direct relationship between the effects of reward in target enhancement and in distractor interference, the magnitude of reward-based target enhancement (high vs. low targets) and distractor interference (low vs. high distractors) was correlated. The magnitude of reward effect was calculated for each participant by averaging the RT differences between high and low reward targets (or distractors) in each set size. There was a significant positive correlation between the two $(\mathrm{r}=.52, p<.001)$ (Fig. $4 d$ ), indicating that the degree of reward-based target enhancement predicts the extent of reward-based distractor interference. The direct relationship observed suggests that a reward-based priority map is influenced by values associated with both target and distractors.

\section{Discussion}

Here, we demonstrate that the effect of reward contingency trained in a bottom-up search task is transferred to a subsequent top-down search task, even though the reward contingency is no longer relevant. It was observed that salience of the target in a top-down search task was selectively enhanced, when it contained a feature that had been associated with high amount of reward in the previous bottom-up search task. Importantly, transfer of the reward effect across different modes of attention cannot be ascribed to participants' persistent intentional strategy. First, subjects were not given explicit instructions as to the reward contingency, and had to learn it implicitly through bottom-up search trials with ambiguous probabilistic reward schedules. Second, we used conjunction search task to engage top-down attention, in which a feature previously associated with reward has to be integrated with a new feature. Therefore, even if subjects were aware of the contingencies of a single feature, it alone cannot fully explain a more efficient search for conjunction targets in different contexts. Third, various set sizes enabled measuring not only absolute RTs but also the slopes of the RTs, and subjects showed shallower search slopes as well as faster absolute RTs for previously high-rewarded targets. While absolute RT differences might be explained by lingering deliberate strategy, the search slope differences cannot be explained by this factor.

Our study provides two important novel findings that elucidate how a reward-based priority map is constructed. First, reward-based transfer influences search efficiency not only by enhancing target salience but also by changing distractor filtering. The presence of previously rewarded distractors either enhanced or interfered with search efficiency depending on whether distractors shared a critical feature with the target, and whether a target template could be set in advance. This result dovetails nicely with previous research (Anderson, et al., 2011a, 2011b) demonstrating that a single previously rewarded distractor captures attention hindering search performance, complemented with a novel finding that previously rewarded distractors, under certain circumstances, enhance search efficiency via increased distractor filtering. The top-down nature of the conjunction search task and the target prime presented before each search display helped participants selectively enhance the target feature and suppress the distractor feature. Also, presence of multiple (instead of single) rewarded distractors could have enabled easier grouping and filtering. Second, when distractors previously associated with reward capture attention, the extent of interference is dependent on the current target template. Previously high-rewarded distractors did interfere with search performance when searching for previously low-rewarded targets, but did not interfere with search when searching for previously high-rewarded targets. These results indicate that not only the reward associations of target and distractors but also the relationship between the two is considered when constructing a reward-based priority map. 
To conclude, our results strongly suggest that top-down and bottom-up attentional selections are both guided by a common priority map based on reward information, rather than by two separate priority maps. Many computational and neurobiological models of attention have provided evidence for the existence of a priority map, a topographic map that represents salience of the sensory input (Fecteau \& Munoz, 2006; Goldberg, Bisley, Powell, \& Gottlieb, 2006; Itti \& Koch, 2001). It is proposed that the multiple maps of different features are combined into a single map, such that the most conspicuous locations in the scene are processed rapidly and efficiently. Although such priority map was originally reflective of bottom-up salience, many follow-up studies demonstrated that top-down influences such as context and goal are also incorporated (Gottlieb, 2007; Mazer \& Gallant, 2003; Thompson $\&$ Bichot, 2005). Within the reward domain, recent studies similarly provided evidence for existence of a topographic map that encodes reward and decision information (Platt \& Glimcher, 1999; Serences, 2008; Sugrue, Corrado, \& Newsome, 2004). Our findings suggesting that the reward-based priority map is commonly used for both bottom-up and top-down attentional guidance add critical evidence to a recently growing body of work that reward- and attention-based selections are integrated (Awh, Belopolsky, \& Theeuwes, 2012; Gottlieb \& Balan, 2010).

\section{Supplementary Material}

Refer to Web version on PubMed Central for supplementary material.

\section{Acknowledgments}

This work was supported by grants from the National Science Foundation (BCS-1059523) and National Institute of Health (R21-EY021644) to S.S.

\section{References}

Anderson BA, Laurent PA, Yantis S. Learned value magnifies salience-based attentional capture. PLoS One. 2011a; 6(11):e27926. PONE-D-11-17766 [pii]. 10.1371/journal.pone.0027926 [PubMed: 22132170]

Anderson BA, Laurent PA, Yantis S. Value-driven attentional capture. Proc Natl Acad Sci U S A. 2011b; 108(25):10367-10371. 1104047108 [pii]. 10.1073/pnas.1104047108 [PubMed: 21646524]

Awh E, Belopolsky AV, Theeuwes J. Top-down versus bottom-up attentional control: a failed theoretical dichotomy. Trends Cogn Sci. 2012; 16(8):437-443. S1364-6613(12)00148-9 [pii]. 10.1016/j.tics.2012.06.010 [PubMed: 22795563]

Corbetta M, Shulman GL. Control of goal-directed and stimulus-driven attention in the brain. Nat Rev Neurosci. 2002; 3(3):201-215. [PubMed: 11994752]

Della Libera C, Chelazzi L. Visual selective attention and the effects of monetary rewards. Psychol Sci. 2006; 17(3):222-227. PSCI1689 [pii]. 10.1111/j.1467-9280.2006.01689.x [PubMed: 16507062]

Della Libera C, Chelazzi L. Learning to attend and to ignore is a matter of gains and losses. Psychol Sci. 2009; 20(6):778-784. PSCI2360 [pii]. 10.1111/j.1467-9280.2009.02360.x [PubMed: 19422618]

Fecteau JH, Munoz DP. Salience, relevance, and firing: a priority map for target selection. Trends Cogn Sci. 2006; 10(8):382-390. [PubMed: 16843702]

Folk CL, Remington RW, Johnston JC. Involuntary covert orienting is contingent on attentional control settings. Journal of Experimental Psychology: Human Perception and Performance. 1992; 18(4):1030-1044. [PubMed: 1431742]

Goldberg ME, Bisley JW, Powell KD, Gottlieb J. Saccades, salience and attention: the role of the lateral intraparietal area in visual behavior. [Review]. Progress in Brain Research. 2006; 155:157175.10.1016/S0079-6123(06)55010-1 [PubMed: 17027387] 
Gottlieb J. From Thought to Action: The Parietal Cortex as a Bridge between Perception, Action, and Cognition. Neuron. 2007; 53(1):9-16. [PubMed: 17196526]

Gottlieb J, Balan P. Attention as a decision in information space. Trends in cognitive sciences. 2010; 14(6):240-248. [PubMed: 20399701]

Humphreys GW, Quinlan PT, Riddoch MJ. Grouping processes in visual search: effects with singleand combined-feature targets. J Exp Psychol Gen. 1989; 118(3):258-279. [PubMed: 2527950]

Itti L, Koch C. Computational modelling of visual attention. Nat Rev Neurosci. 2001; 2(3):194-203. [PubMed: 11256080]

Kastner S, Ungerleider LG. Mechanisms of visual attention in the human cortex. Annu Rev Neurosci. 2000; 23:315-341. [PubMed: 10845067]

Kiss M, Driver J, Eimer M. Reward priority of visual target singletons modulates event-related potential signatures of attentional selection. Psychol Sci. 2009; 20(2):245-251. [PubMed: 19175756]

Kristjansson A, Sigurjonsdottir O, Driver J. Fortune and reversals of fortune in visual search: Reward contingencies for pop-out targets affect search efficiency and target repetition effects. Atten Percept Psychophys. 2010; 72(5):1229-1236. 72/5/1229 [pii]. 10.3758/APP.72.5.1229 [PubMed: 20601703]

Lee J, Shomstein S. The differential effects of reward on space- and object-based attentional allocation. J Neurosci. 2013; 33(26):10625-10633. 33/26/10625 [pii]. 10.1523/JNEUROSCI. 5575-12.2013 [PubMed: 23804086]

Mazer JA, Gallant JL. Goal-Related Activity in V4 during Free Viewing Visual Search: Evidence for a Ventral Stream Visual Salience Map. Neuron. 2003; 40(6):1241-1250. [PubMed: 14687556]

Navalpakkam V, Koch C, Rangel A, Perona P. Optimal reward harvesting in complex perceptual environments. Proc Natl Acad Sci U S A. 2010; 107(11):5232-5237. 0911972107 [pii]. 10.1073/ pnas.0911972107 [PubMed: 20194768]

Nothdurft HC. The role of features in preattentive vision: Comparison of orientation, motion and color cues. Vision Research. 1993; 33(14):1937-1958.10.1016/0042-6989(93)90020-w [PubMed: 8249312]

Platt ML, Glimcher PW. Neural correlates of decision variables in parietal cortex. [10.1038/22268]. Nature. 1999; 400(6741):233-238. [PubMed: 10421364]

Serences JT. Value-based modulations in human visual cortex. Neuron. 2008; 60(6):1169-1181. [PubMed: 19109919]

Shomstein S, Johnson J. Shaping attention with reward: Effects of reward on space- and object-based selection. Psychological Science. in press.

Shomstein S, Lee J, Behrmann M. Top-down and bottom-up attentional guidance: investigating the role of the dorsal and ventral parietal cortices. Exp Brain Res. 2010; 206(2):197-208.10.1007/ s00221-010-2326-z [PubMed: 20571784]

Sugrue LP, Corrado GS, Newsome WT. Matching Behavior and the Representation of Value in the Parietal Cortex. Science. 2004; 304(5678):1782-1787.10.1126/science.1094765 [PubMed: 15205529]

Thompson KG, Bichot NP. A visual salience map in the primate frontal eye field. Prog Brain Res. 2005; 147:251-262. citeulike-article-id:665631. [PubMed: 15581711]

Treisman A, Gelade G. A feature-integration theory of attention. Cognit Psychol. 1980; 12(1):97-136. [PubMed: 7351125]

Wolfe JM. Guided Search 2.0: A revised model of visual search. Psychonomic Bulletin \& Review. 1994; 1(2):202-238. citeulike-article-id:80546. [PubMed: 24203471] 
a

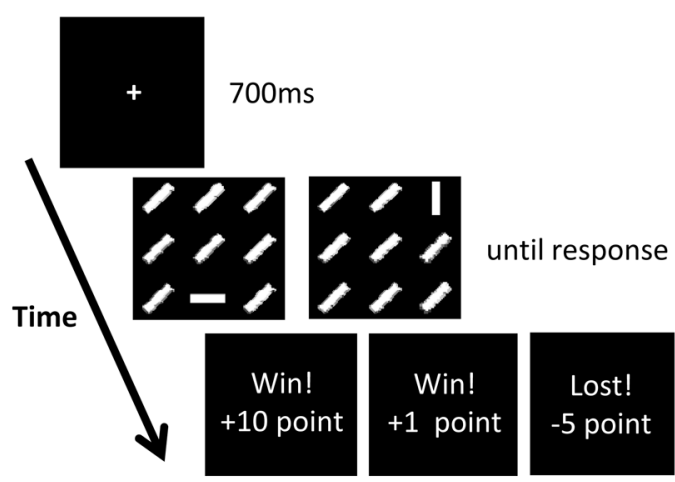

b

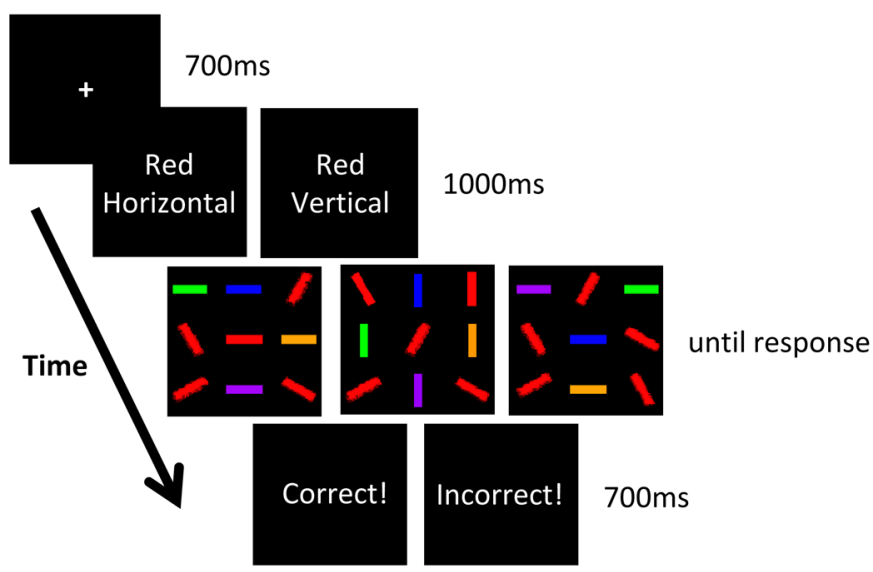

Figure 1.

Sequence of events and time course for a trial during the (a) pop-out search task and (b) conjunction search task. 
a

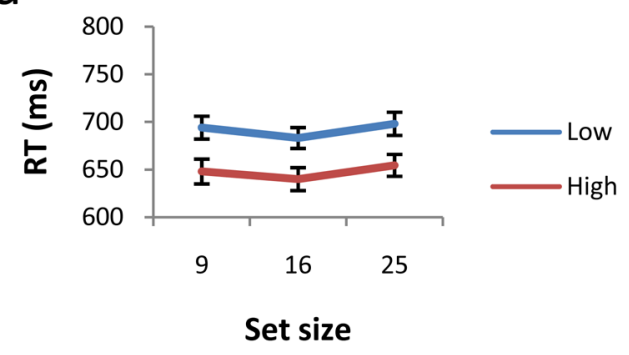

C

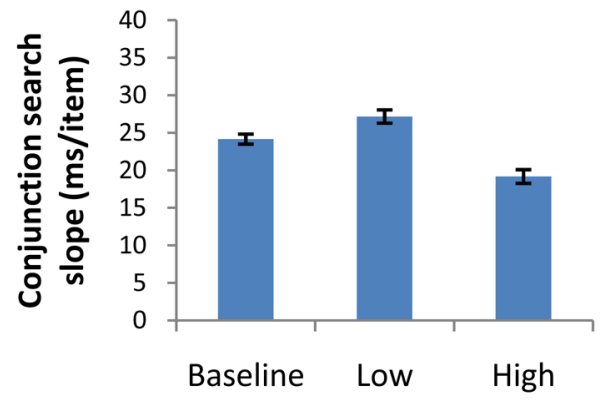

b

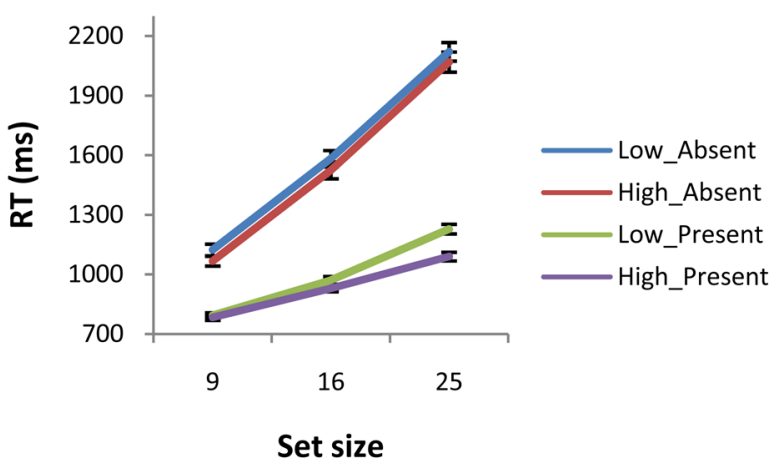

d

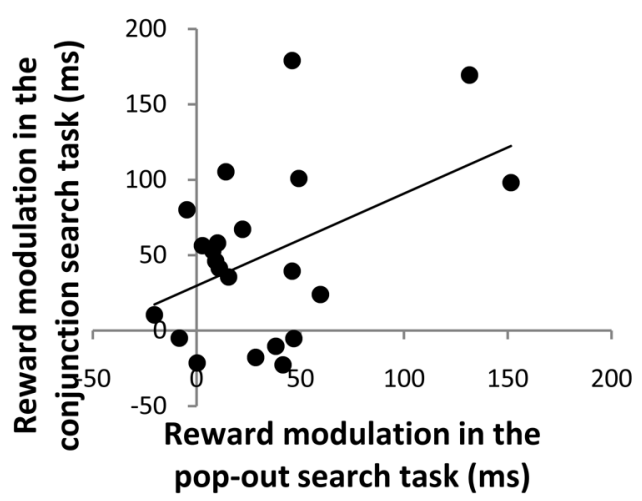

Figure 2.

(a) Mean RTs for high and low reward targets for each set size in the pop-out search task in Experiment 1. (b) Mean RTs for each condition (a combination of reward level and target presence) for each set size in the conjunction search task in Experiment 1. (c) Mean conjunction search slopes obtained before reward training (baseline) and after reward training for previously high-rewarded and low-rewarded targets (d) A scatterplot illustrating the relationship between the magnitude of reward effects in the pop-out search task and in the conjunction search task. Here, and in all figures, error bars represent standard error of the mean (SEM). 
a
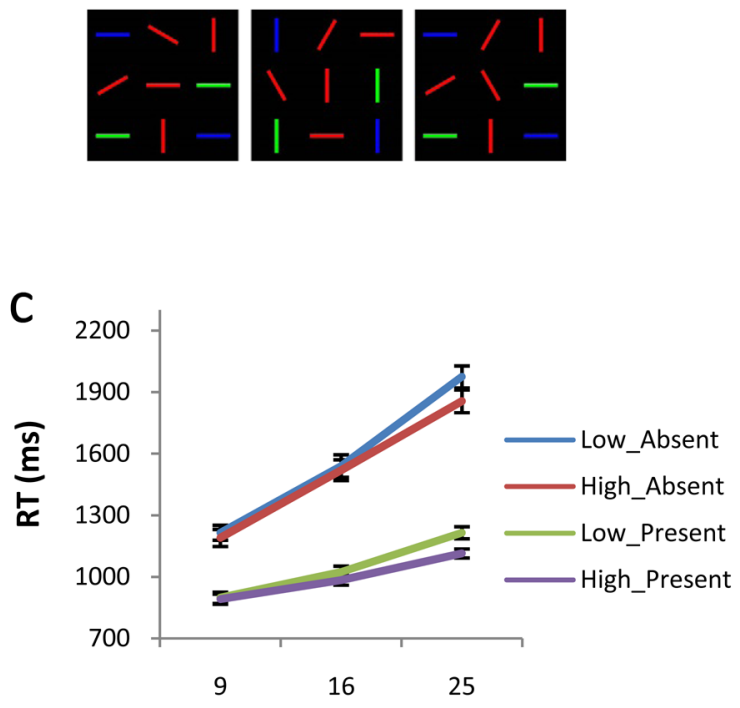

Set size

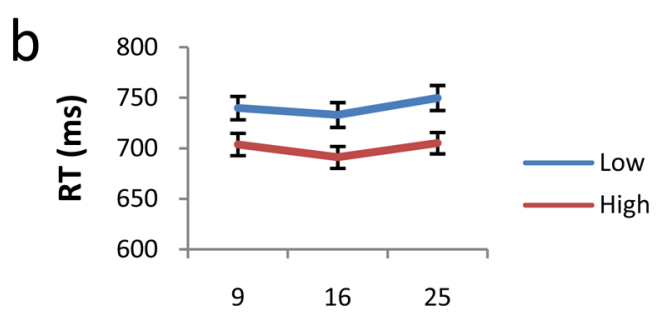

Set size

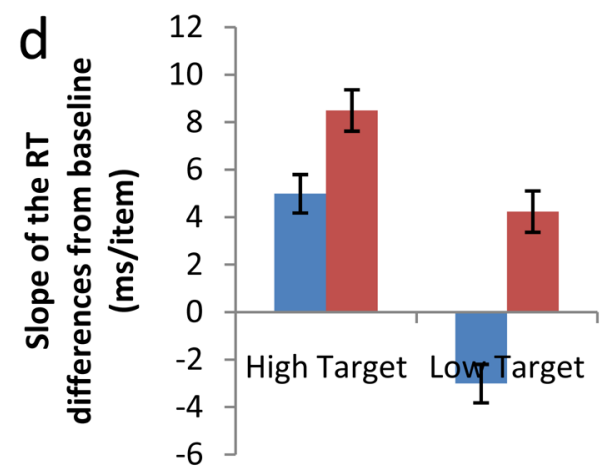

Neutral Dist.

(Exp.1)

Rewarded Dist.

(Exp.2)

Figure 3.

(a) Examples of conjunction search displays in Experiment 2 for a red horizontal target, red vertical target, and target-absent trial, respectively. Half of the target-colored (red)

distractors had an orientation that was previously associated with reward, and the other half of the red distractors had neutral orientations. (b) Mean RTs for high and low reward targets for each set size in the pop-out search task in Experiment 2. (c) Mean RTs for each condition (a combination of reward level and target presence) for each set size in the conjunction search task in Experiment 2. (d) Slope of the RT differences from baseline (ms/ item) of previously high- and low-rewarded targets among neutral distractors (Exp.1) or among previously rewarded distractors (Exp.2). 

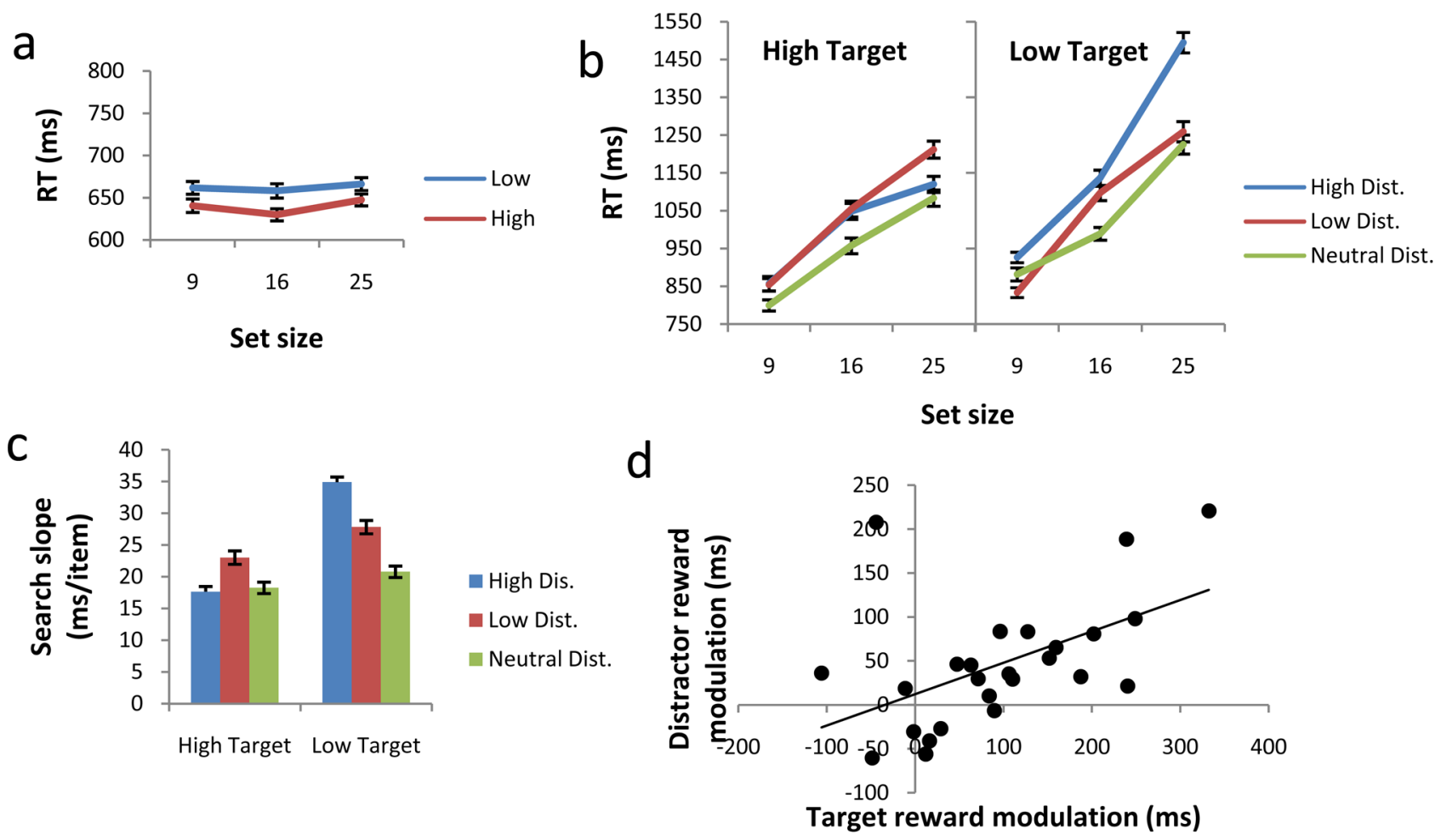

Figure 4.

(a) Mean RTs for high and low reward targets for each set size in the pop-out search task in Experiment 3. (b) Mean RTs for each condition (a combination of target and distractor's reward levels) for each set size in the conjunction search task in Experiment 3. (c) Search slope (ms/item) of each condition (a combination of target and distractor's reward levels) in Experiment 3. (d) A scatterplot illustrating the relationship between the magnitude of reward effects in target enhancement (High vs. Low targets) and in distractor interference (Low vs. High distractors). As the reward effects in target enhancement increased, the reward effects in distractor interference also increased. 